
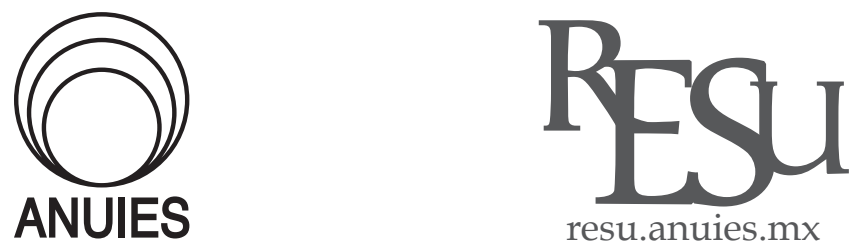

ARTíCULO

\title{
La difusión de la ciencia en México a través de artículos científicos. Condiciones y contextos
}

\section{The diffusion of science in Mexico through scientific papers. Conditions and contexts}

\author{
Santos López-Leyva*, Aída Alvarado-Borrego ${ }^{* *}$ y Ana Bárbara Mungaray-Moctezuma*** \\ * Profesor e investigador de la Facultad de Economía y Relaciones Internacionales de la Universidad Autónoma \\ de Baja California, campus Tijuana. Correo electrónico: slleyva@uabc.edu.mx \\ * Profesora e investigadora de la Universidad de Occidente, campus Los Mochis, Sinaloa. \\ * Profesora e investigadora de la Facultad de Economía y Relaciones Internacionales de la Universidad Autónoma \\ de Baja California, campus Tijuana.
}

Recibido el 02 de marzo de 2018; aceptado el 29 de octubre de 2018.

\section{Resumen}

Este trabajo examina la producción de investigadores mexicanos. Analiza el comportamiento de publicaciones mexicanas en los bancos de información scopus y Web of Science. México en la ciencia mundial, en número de artículos, ha crecido, pero no así en indicadores de calidad. Se aplicó un cuestionario a 373 investigadores donde se identificó que la principal razón de ellos para publicar tiene que ver con el fortalecimiento de la ciencia y las instituciones. Señalan de igual modo que las universidades deben instrumentar talleres de publicación en los posgrados, pues la falta de tiempo para investigar y escribir es la principal barrera en la publicación. Los investigadores conocen revistas para publicar.

Palabras clave: Artículo científico; Difusión científica; Revisión por pares; Investigación científica; Investigador académico. 


\begin{abstract}
This paper examines the production of Mexican researchers and it analyzes the behavior of Mexican publications in the information banks scopus and Web of Science. Mexico has grown in world science in number of articles but, not in quality indicators. A questionnaire was applied to 373 researchers where the main reason for researchers to publish is the strengthening of science and institutions; they point out that universities should implement workshops with graduate students; lack of time to research and write is the main barrier in the publication. The researchers know journals for their publications.
\end{abstract}

Keywords: Scientific paper; Scientific diffusion; Peer review; Scientific research; Academic researcher.

\title{
Introducción
}

T a difusión de la ciencia a través de la publicación de artículos científiـos es una actividad por medio de la cual los investigadores académicos dan a conocer sus trabajos. Las instituciones de educación superior y los centros de investigación se preocupan porque sus académicos alcancen mejores indicadores de productividad a través de las publicaciones en revistas científicas indexadas. Los diferentes organismos relacionados con el impulso a la ciencia promueven la investigación y la difusión de sus resultados con el fin de mejorar el nivel científico del país, y todo ello hace que la participación de México en la ciencia mundial mejore cada día.

Este trabajo presenta un panorama acerca de la publicación de artículos por parte de investigadores mexicanos, conforme su propósito es conocer cuál es el comportamiento de las publicaciones mexicanas en la ciencia mundial en cuanto a su monto y su impacto. Un segundo objetivo es indagar acerca de las condiciones que tienen los científicos mexicanos para la realización de sus publicaciones. Se parte de la hipótesis de que la participación de México en la ciencia mundial ha crecido, esto tomando en consideración la cantidad de publicaciones arbitradas realizadas por investigadores mexicanos en las múltiples revistas científicas indexadas que se publican a nivel mundial.

Para conocer lo anterior se llevó a cabo la revisión de múltiples documentos que exponen resultados acerca de la difusión de la ciencia en México; además se aplicó un cuestionario a 373 académicos de universidades públicas en el país. 


\section{La necesidad de publicar y factores limitantes}

Los investigadores académicos tienen la necesidad de publicar los resultados de sus trabajos, para lo cual existen múltiples razones. Cargill y O'Connor (2009) apuntan varias de ellas: por este medio, los académicos comparten ideas y resultados con sus colegas; dejan constancia escrita de sus trabajos de investigación, que al sumarlos a otros, fortalecen el monto de conocimientos de una sociedad; reciben reconocimiento de parte de la comunidad académica a sus ideas y resultados; atraen el interés de otros académicos hacia sus proyectos y área de conocimiento; toman retroalimentación para el mejoramiento de sus trabajos; legitiman su investigación; tienen acceso a revistas importantes donde investigadores de su mismo campo de interés publican y exponen sus experiencias; reciben correspondencia de diferentes partes del mundo; desarrollan habilidades para la búsqueda de información y presentación de la misma. Claudio (2016:18) dice que son ocho las principales razones que tiene un académico para realizar una publicación científica: 1) poner sus resultados en papel ayuda a entender las metas de la investigación, permite interpretar los datos de mejor manera y facilita comparar su trabajo con otros; 2) la revisión por pares brinda una importante retroalimentación que posibilita validar la investigación y mejorar las siguientes etapas del trabajo; 3) comunicar la información encontrada ayuda a otros investigadores a avanzar en su trabajo y fortalecer el conocimiento existente en el campo; 4) poner la investigación en contexto define núcleos y fronteras de acción en un campo del conocimiento; 5) mejora el entendimiento público acerca del tema de investigación; 6) permite avanzar en la carrera académica a través de reconocimientos y promociones; 7) ayuda a convertirse en un experto en el campo del conocimiento: 8) la evaluación por pares brinda evidencia para tomar decisiones en cuanto a fondos de investigación. Estas razones, Debnath y Venkatesh (2015: 107) las resumen en cinco puntos: 1) la publicación brinda la oportunidad de compartir pensamientos e ideas con el resto de la comunidad científica, quien ayuda a mejorar la perspectiva científica del artículo y el nivel de conocimiento en él comprendido; 2) ayuda a difundir el conocimiento entre los pares académicos; 3) proporciona un sentido de responsabilidad cumplida ante la sociedad de parte del investigador; 4) impulsa la formación y la carrera del investigador; 5) apuntala el crecimiento de la ciencia ya que el futuro de ésta depende, en gran parte, de la difusión de las contribuciones científicas. En México existen motivaciones especiales para lograr una publicación, entre ellas, los investigadores publican para mejorar el puntaje en los estímulos en sus universidades, para integrarse y permanecer en el Sistema Nacional de Investigadores (SNI), lograr el grado de profesor con perfil de- 
seable del Programa de Desarrollo Profesional Docente (Prodep); mejorar el grado de consolidación de los cuerpos académicos y buscar la acreditación de los programas de académicos donde laboran.

En la actualidad los diferentes organismos e instituciones encargados de evaluar la producción de conocimiento privilegian el artículo científico sobre otras formas utilizadas en la difusión de la ciencia, esto por varios motivos: los resultados de la investigación se muestran a la comunidad académica que tiene la posibilidad de opinar en torno a los mismos; la publicación de un artículo recorre un proceso de evaluación por pares que mejora la calidad científica del mismo; se generan indicadores de citación que vigorizan la pertinencia del reporte; se establecen comparaciones en el contexto mundial acerca del contenido del trabajo; crea una reserva de conocimiento que se comparte con la comunidad académica; incrementa la visibilidad del conocimiento; funciona como mecanismo de entrenamiento para árbitros, editores y autores; fortalece el proceso de enseñanza de la ciencia y apoya la promoción de su campo disciplinar (Cargill y O’Connor, 2009; Sabaj, 2009; López-Leyva, 2013). También fortalece el trabajo conjunto de los equipos académicos, puede dibujar el perfil científico de un país y ayuda a definir una política en ciencia y tecnología.

En el campo internacional, los diferentes rankings de universidades consideran la publicación y la citación como principales elementos a evaluar; así, en el Academic Ranking World Universities (ARWU), al menos el 60\% del puntaje lo basa en estas actividades al establecer los siguientes porcentajes en su marco de referencia: investigadores altamente citados en 21 categorías temáticas diferentes, 20\%; artículos publicados en las revistas Nature y Science, 20\%; artículos publicados en las revistas del Índice de Citas Expandido y en el Índice de Citas en Ciencias Sociales de la Web of Science, 20\%. Por lo tanto, el 60\% del peso de este ranking se ubica en las publicaciones; cabe aclarar que otros indicadores también están relacionados con dicha actividad, como los premios Nobel y Medallas Fields obtenidas por los académicos y ex alumnos de las universidades. El ranking mundial del Suplemento del Times se calcula sobre trece indicadores, donde las citas aportan el 30\% del valor del ranking y la productividad en la investigación aporta el 6\%.

En México, los organismos evaluadores de la investigación insisten cada vez más en la necesidad de publicar artículos científicos, así el artículo 31 del reglamento del SNI establece, en primer lugar: "[...] artículos que hayan sido sujetos a un arbitraje riguroso por comités editoriales de reconocido prestigio" (Conacyt, 2017a), este constituye el primer indicador de productividad de los investigadores. También los marcos de referencia y criterios de cada una de las siete áreas de conocimiento de SNI consideran al artículo científico como indicador para la evaluación, aunque cada una de ellas lo asume de diferente manera (Conacyt, 2017b). 
Área I. Matemáticas y Ciencias de la Tierra. Como indicador señala que sólo se tomarán en cuenta los artículos publicados en revistas de alto nivel académico internacional, indexadas e incluidas en SCI (Science Citation Index) o en el Padrón de Excelencia del Conacyt y que además no incluya a ninguno de los autores del artículo citado. Se considerará el factor de impacto de las revistas para ponderar la calidad del artículo publicado y sus citas respectivas.

Área II. Biología y Química. El primer elemento a evaluar lo constituye artículos de investigación en revistas especializadas de calidad internacional, con arbitraje riguroso que estén indexadas con un factor de impacto publicado estrictamente en el "Journal Citation Reports" (JCR).

Área III. Medicina y Ciencias de la Salud. Considera como productos de investigación los artículos científicos originales publicados en revistas que estén incluidas en el Journal Citation Reports, en sus dos versiones (Science Citation Index Expanded y Social Science Citation Index).

Área Iv: Humanidades y Ciencias de la Conducta. Considera en primer lugar los libros y después los artículos en revistas con arbitraje riguroso.

Área v. Ciencias Sociales. Artículos de investigación en revistas especializadas, de calidad nacional e internacional, con arbitraje. Se dará preferencia a las revistas indizadas en el caso de las internacionales y del padrón del Conacyt en el caso de las nacionales.

Área vi. Biotecnología y Ciencias Agropecuarias. Publicación de trabajos de investigación en revistas científicas con arbitraje e impacto internacional, incluidas en el Journal Citation Reports (JCR) del ISI y en el Índice de Revistas Mexicanas de Investigación Científica y Tecnológica del Conacyt. Cabe aclarar que el ISI ahora es la Web of Science y el índice del Conacyt se ha trasformado en "Revistas arbitradas del Conacyt".

Área VII. Ingenierías. Artículos en revistas indizadas en índices del mayor prestigio. Artículos en revistas del Padrón del Conacyt.

Como se puede observar, cinco áreas establecen como indicador principal los artículos publicados en revistas con factor de impacto y que aparezcan en JCR; sólo las áreas IV y v no consideran este elemento, establecen los artículos sólo en revistas arbitradas.

El artículo científico es un escrito que presenta y discute los resultados de un proyecto de investigación académica, concluida o en proceso, que después de pasar por una rigurosa evaluación por parte de especialistas se considera como una contribución original y significativa para el desarrollo de un campo del conocimiento científico (Sabaj, 2009; López-Leyva, 2013; Katz, 1985, Day y Gastel, 2006).

Para que el proceso de investigación se considere completo es necesario comunicar los resultados encontrados. Como dice Toworfe (2012), no basta con lle- 
var a cabo una excelente investigación que arroje resultados relevantes, sino que es necesario realizar una buena comunicación de los mismos. Dice el portal de la Red de Revistas Científicas de América Latina y El Caribe, España y Portugal (Redalyc): "Ciencia que no se ve, no existe".

Según el Manual de la American Psychological Association (APA) (APA, 2010a y APA 2010b), existen diferentes tipos de artículos: estudios empíricos, reseñas, artículos teóricos, artículos metodológicos y estudios de caso. Belcher (2009) enumera catorce tipos de publicaciones académicas: bibliografía comentada, reseñas de libros, artículos profesionales, notas, entrevistas, traducciones, artículo de respuesta, artículo de revisión, artículo teórico, artículo en ciencias sociales, artículo con enfoque cualitativo, artículo con enfoque cuantitativo, una investigación interpretativa, artículo en humanidades y artículo en ciencias naturales. Por su parte, Holst (2015) presenta otra lista de trabajos que publican las revistas científicas: cartas o comunicaciones cortas, artículos completos, notas, revisiones, reseñas, comentarios técnicos, correcciones y aclaraciones.

Para la publicación de un artículo científico se tienen varios obstáculos, uno de ellos es lo que Belcher (2009) denomina disfunción en la escritura en la academia, para lo cual recurre a una encuesta (Lindholm et. al 2005 citado en Belcher, 2009) que comprendió a 40000 académicos de universidades de Estados Unidos que arrojó que el $26 \%$ de los profesores invierten cero horas a la semana en la escritura, al menos $27 \%$ nunca ha publicado un artículo en una revista científica, $43 \%$ no ha publicado nada en los últimos tres años, $62 \%$ nunca ha publicado un libro y sólo el $28 \%$ ha producido más de dos publicaciones en los últimos dos años

La misma autora (Belcher, 2009) señala 28 limitantes en la escritura de un artículo científico, de las cuales seis de ellas están relacionadas con el tiempo disponible para escribir, diecinueve con elementos de tipo personal y sólo tres con la estructura y falta de asesoría. También establece que algunos académicos no publican porque piensan que sólo lo serán aquellos artículos que: a) cuentan con una gran revisión teórica, b) que integran ideas muy interesantes y c) los que son enteramente originales y ellos no disponen de las condiciones para producir un trabajo con estas características.

Cargill y O'Connor (2009) apuntan que existe una serie de dificultades para lograr una publicación: en primer lugar se requiere poseer habilidades especiales para la investigación y la escritura, pero, además, no todos los resultados de investigación son nuevos y tienen interés científico; los experimentos y proyectos no siempre trabajan en forma adecuada para concluir en una publicación de calidad; los resultados positivos y claros son siempre fáciles de publicar, pero no todos presumen estas características; las revistas científicas tienen requerimien- 
tos especiales difíciles de cumplir, y por este medio el investigador se abre a las críticas a las que algunos investigadores no muestran disponibilidad.

Una limitante, de la que no se ha hablado, es la concerniente a la dificultad para escribir, pero además del complejo proceso que encierra enseñar esta técnica en el posgrado. En cuanto a las dificultades para la enseñanza de la escritura científica a los estudiantes de Posgrado, Espino (2015) establece que se enfrentan problemas en cuatro áreas fundamentales: 1) para seguir una estructura canónica acorde a la comunidad científico-académica de la que forman parte; 2) para utilizar un estilo de redacción formal; 3) para dar coherencia y cohesión a la presentación del contenido sobre los que se escribe; 4) para citar fuentes bibliográficas durante el proceso. A lo anterior es importante agregar que el principal problema que enfrenta el profesor en los seminarios de tesis es la definición del objeto de estudio, como dice Difabio de Anglat (2011: 945): “[...] la delimitación del objeto de estudio demanda mucho tiempo porque son contados los doctorantes que tienen claro qué quieren estudiar".

\section{Métodos y datos}

Como primera actividad se llevó a cabo una revisión de la participación de México en los dos bancos de información científica de mayor tamaño existentes en el mundo, que son la Web of Science (WoS) y scopus. Los datos de interés giran alrededor de la participación de México en el monto de publicaciones mundiales y el impacto que presentan estas publicaciones a través del número de citas, el índice $\mathrm{H}$ de Hirsh y el Factor de Impacto

Para conocer las condiciones que exponen los profesores bajo las cuales se realiza la publicación, se aplicó un cuestionario de nueve preguntas a 373 académicos, de los cuales fueron 236 profesores con el grado de doctor y 137 estudiantes de doctorado, de las siguientes universidades: Universidad Autónoma de Baja California (UABC), 144 cuestionarios; Universidad Autónoma de Sinaloa (UAS), 139 cuestionarios; Benemérita Universidad Autónoma de Puebla (BUAP), 86 cuestionarios; Universidad de Occidente (U de O), 21 cuestionarios; y 1 para las universidades de Zacatecas y Colima. La información anterior proviene de las dos primeras preguntas del cuestionario. La tercera estuvo referida al área de conocimiento, donde los cuestionarios se distribuyeron de la siguiente manera: área I, 27 cuestionarios; área II, 15; área III, 16; área IV, 66; área V, 169; área VI, 26; y área VI, 54. Como se puede observar, la mayoría de los informantes pertenece al área v de Ciencias Sociales, al alcanzar el 45\% de los cuestionarios, y sigue el área IV con el 18\%; entre éstas dos alcanzan el $63 \%$ de los encuestados. Para los 
casos de la UABC, UAS y U de O, el cuestionario se aplicó totalmente al azar a profesores que se encontraban en los cubículos a la hora de la visita a una facultad o departamento, y para la BUAP, el cuestionario se aplicó en un taller de trabajo sobre difusión de la ciencia.

La cuarta pregunta del cuestionario se encargó de conocer las razones que esgrimen los profesores para emprender la escritura y la publicación de un artículo, donde 240 encuestados seleccionaron la necesidad de difusión de la ciencia; en segundo lugar, para mejorar su curriculum; y en tercero, para el mejoramiento de los vínculos con otros investigadores. En el campo de otros, se recibieron 30 opiniones, donde destaca el mejoramiento de la ciencia y el fortalecimiento institucional, para lo cual se ofrecieron trece sugerencias. Un segundo grupo se refiere al mejoramiento personal con 5 opiniones; también se tuvieron cinco juicios en torno a que se ven forzados por la institución y por la reglamentación existente en educación superior; cuatro señalaron que sólo lo hacen por interés y disfrute personal, y tres en torno a la búsqueda de solución de problemas nacionales.

La siguiente cuestión estuvo referida a la existencia de políticas para la publicación de los resultados de investigación; 220 (59\%) profesores señalaron que existen dichas políticas, 66 (18\%) expresaron que no existen y 87 (23\%) dijeron no conocer la existencia de esas políticas. En el renglón de otras se recogió la opinión de 78 investigadores, las cuales se distribuyeron en cinco grupos: 1) la institución cuenta con políticas para la publicación, 37 opiniones; 2) no cuenta con políticas, 21;3) carece de recursos, 10; 4) sólo es disposición personal, 5; y 5) factores de carácter político impiden la publicación, 2.

La pregunta 6 indaga si en el programa de posgrado donde realizaron o realizan sus estudios se desarrolla algún tipo de taller o curso para impulsar la publicación de artículos científicos; 167 encuestados dijeron que sí y 206 que no. Se recibieron 85 opiniones de los investigadores, las cuales se agruparon en cuatro rubros: 1) existencia de cursos no permanentes - además, no aparecen en el plan de estudios - , 33 opiniones; 2) no existe un taller, 24; 3) sería muy importante recibirlo, 9; y 4) este tipo de talleres se toman por iniciativa personal, 7.

Entre las dificultades que enfrentan para publicar, que fue el cuestionamiento de la pregunta número 7 , los profesores dijeron que la mayor limitante es la falta de tiempo, pues 180 maestros la pusieron en primer lugar, correspondiendo al $48 \%$ del total; la siguiente fue la falta de asesoría con 85 frecuencias correspondiente al 23\%, y la falta de resultados de investigación 58 frecuencias (15.5\%). Le siguieron falta de espacios adecuados, 27; carencia de bibliografía 17, desconocimiento de revistas con sólo 6 resultados. En otras, se recogieron 62 opiniones, de las cuales 19 marcaron la carencia de apoyo económico; 15 insistieron en la falta de tiempo para la publicación; 14 reconocieron la carencia de capacidad 
técnica que existe entre los investigadores para la publicación; 10 a la carencia de políticas institucionales para promover la difusión de la ciencia; y 4 al desconocimiento de las revistas

Después se invitó a los investigadores a sugerir medidas para mejorar la publicación de artículos en su institución. Se tuvieron 245 respuestas que corresponde al 89.74\% del total de la muestra. Estas sugerencias se agruparon de la manera siguiente: 1) dedicar más recursos económicos a la investigación y difusión de sus resultados, 72 sugerencias $(29.4 \%)$; 2) promover asesoría, cursos y talleres para mejorar la publicación, 68 sugerencias $(27.7 \%)$; 3) apoyo en infraestructura para la investigación, 41 sugerencias 16.7\%; 4) definir políticas de difusión de la ciencia para la universidad a nivel general, 26 sugerencias (10.6\%); $5)$ establecer mecanismos para descarga en docencia para dedicar tiempo a la investigación, 14 sugerencias (5.7\%); y 6) dijeron desconocer la existencia de políticas, 9 sugerencias.

En cuanto a las revistas, la pregunta la respondieron 334 profesores y 39 no contestaron. 31 no señalaron ninguna revista, sólo los bancos de información como Redalyc y SciELO; se hizo una lista de 334 revistas y como solamente 303 investigadores sugirieron revistas, esto implica que 31 investigadores lo hicieron con dos revistas.

\section{Análisis y discusión de resultados}

Atendiendo a las estadísticas de Scimago Country Rank que trabaja con datos de Scopus, México ha incrementado su participación en la ciencia mundial considerando el número de artículos científicos publicados por sus investigadores; el país pasó de participar con el $0.50 \%$ de la ciencia mundial en el año 2000, al 0.71\% en 2014, pero su contribución en América Latina ha disminuido del $19.25 \%$ en el 2000 al $16.2 \%$ en 2014, lo cual implica una baja de tres puntos porcentuales como resultado de la dinámica seguida por Brasil que incrementó su participación en la ciencia tanto a nivel mundial como de América Latina. En el 2000, los investigadores mexicanos publicaron 6133 artículos, y en 2014, 16 607, lo que representa una tasa aproximada de crecimiento del $7 \%$ anual. Por otra parte, la Web of Science señala que en una década - de 2003 a 2012 - el número de artículos aumentó en un 65\% y su participación en la ciencia mundial creció de un $0.7 \%$ a un $0.8 \%$; el factor de impacto mejoró en un 36\%, sin embargo en este indicador sigue estando abajo del promedio mundial en un $2 \%$ en los mismos años. El área con mayor factor de impacto sigue siendo la Astronomía. 
México, en 2016, ocupaba el lugar 29 en la producción de artículos científicos a nivel mundial: alcanzó una citación promedio de 11.04 por artículo en el mismo periodo, con un Índice H=352. Si bien se ubicó en el 29 lugar en la producción de artículos, hubo países con menos producción pero que tuvieron más alta citación y mayor Índice H: Noruega en el número 30, con una citación promedio de 19.25 y H=491; Portugal en el número 31, con una citación de 13.57 y H=379; Singapur en el lugar 32, con una citación promedio de 16.98 y H=454; Hong Kong en el lugar 33, con promedio de 18.19 de citación y H=447. Así, existen varios países con menor número de artículos pero con indicadores más altos, tal es el caso de Sud África.

En cuanto al promedio de citas por artículo, al realizar un ejercicio de integrar 150 países en cinco grupos, en el grupo 1 se ubican aquellos países que tienen más de 21 citas en promedio, con un total de 14 países; grupo 2, de 16 a 20 citas, 28 países; grupo 3, de 11 a 15 citas, 51 países; grupo 4, de 6 a 10 citas, 48 países; y grupo 5, de 1 a 9 citas, 9 países.

México se ubica en el grupo 3, el cual tiene mayor número de países, con valor de citación igual a 11.04; si se hace un ordenamiento de países por citación, México quedaría en el lugar 81, muy lejos del lugar de publicación, lo cual indica que los artículos de los mexicanos requieren mayor penetración en la ciencia mundial. Pero si el ejercicio se realiza considerando el Índice $H$, el país muestra mejores condiciones, pues su $\mathrm{H}=363$ lo ubica en el lugar 33 a nivel mundial, lo cual implica que hay 363 artículos mexicanos que tienen 363 citas como mínimo.

El llevar a cabo una revisión del comportamiento de México en la producción científica con datos de la Web of Science (WoS) se encuentra que de 2008 a 2015 la producción de artículos científicos de nuestro país experimentó una tasa media de crecimiento del $4.92 \%$. Pero la producción científica mexicana ha observado un estancamiento a partir de 2012 y en 2014 y 2015, pues en esos años la producción fue la misma. Si se revisa la participación de México a nivel mundial, se tiene que a partir de 2012 ha alcanzado entre el 0.7 y el 0.8 por ciento. Del 100\% de artículos, la aportación de las áreas es como sigue: Plantas y Animales, 12.62\%; Física, 10.31\%; Medicina Clínica, 9.62\%; Química, 9.58\%; Ingeniería 8.48\%; Ecología y Medio Ambiente, 6.47\%; Agricultura, 5.88\%; Biología y Química, 4.82\%; Ciencias Sociales, $4.4 \%$; Ciencias de los materiales, $4.35 \%$.

En comparación con los países de la Organización Económica para la Cooperación y el Desarrollo (OECD), México se encuentra en el lugar 22, de los 34 países en cuanto a su participación en la ciencia mundial. Es el segundo en América Latina, después de Brasil que tiene una participación del 1.99\%.

Pero si bien la participación de México en cuanto al número de artículos es significativa, no se puede decir lo mismo en cuanto a su calidad y su impacto. Las áreas que tienen el mayor factor de impacto son: Biología molecular y gené- 
tica, Física y Ciencias espaciales. Las tres áreas con menor factor de impacto son Ciencias Sociales, Matemáticas y Economía y Negocios.

Al revisar el Impacto Relativo al Mundo (IRM), o sea la importancia que brinda la comunidad académica mundial a la producción científica de un país, México se ubica en el lugar 33, de los 34 países de OECD con un valor de 0.98; con esta cifra solamente aventaja a Turquía, con un valor de 0.73. En América Latina, es superado por Chile, 1.28; Colombia, 1.22; Argentina, 1.14

En cuanto a las razones que tienen los académicos para publicar, la principal de ellas es la difusión de la ciencia referida por el 64\% de los encuestados; otras están relacionadas con el mejoramiento del curriculum del investigador y la tercera al mejoramiento de las relaciones con otros investigadores. En las diferentes opiniones se aprecia una preocupación por el fortalecimiento de la ciencia y la búsqueda del mejoramiento de las instituciones donde laboran. En el contexto global existen opiniones en torno a que este tipo de intenciones entre los académicos tienden a disminuir, pero en cambio surge fuerte el propósito relacionado con el incremento de puntos para asegurar la permanencia y mejorar su situación en las universidades y centros de investigación. Ortinau (2011) señala que estas últimas son las motivaciones que están impulsando a los académicos, pero en esta dirección sólo se tuvieron cinco opiniones, las cuales también agregan que se ven forzados por la institución y por la reglamentación existente en educación superior. Asimismo, se observaron opiniones concretas en torno a la necesidad de demostrar a la sociedad que el dinero invertido en ciencia está bien aprovechado; se debe buscar mejor proyección de la institución para la que se labora y mejorar los indicadores institucionales. Estas opiniones no concuerdan con lo encontrado por Gómez, Jiménez y Moreles (2014: 172), en torno a la existente simulación entre los académicos porque pocos trabajan y todos se apuntan, y que los académicos se dedican exclusivamente a elegir las actividades que les redituarán mayor número de puntos para mejorar su curriculum. Sin duda, no son justas estas opiniones para los investigadores en México, porque a pesar de las limitaciones han hecho crecer la ciencia mexicana como se ha visto en datos presentados en este documento.

En lo referente a la existencia de políticas de impulso a la publicación en las universidades y centros de investigación, 59\% de los investigadores confirmó la existencia de estas políticas; 37 de ellos ofrecieron su opinión donde se puede resaltar la existencia de las siguientes:

- La exigencia de publicación de artículos en el posgrado.

- La creación de un departamento para apoyar las publicaciones.

- La existencia de políticas, con la necesidad de una mayor difusión de las mismas para que los académicos las conozcan mejor.

- La creación de padrones de investigadores locales e institucionales. 
- El impulso a los cuerpos académicos para que publiquen de manera conjunta.

- Existen políticas pero no se actualizan de cara a las nuevas tendencias, que es publicar en revistas con mayor visibilidad.

- La universidad sigue impulsando la publicación de libros

- Existen políticas que se establecieron en los programas de estímulo económicos, para la obtención de ingreso y permanencia en el SNI, para consolidar cuerpos académicos.

- Un programa de apoyo para cubrir costos de publicación en revistas que lo requieran.

- Promueven políticas para hacer más visibles a los investigadores.

- Promueven la realización de coloquios para la presentación de los avances de investigación y existen incentivos para quienes publican en revistas de alta visibilidad.

De manera general, los doctorados en México todavía no disponen de programas específicos de entrenamiento para que sus estudiantes logren publicaciones en revistas indexadas utilizando los resultados de investigación, aspecto que mencionaron la mayoría de los encuestados. Sólo el $45 \%$ de los investigadores reportó la existencia de cursos específicos que buscan esta capacitación; 33 académicos se refirieron a que existen, pero que hace falta institucionalizarlos, porque ofrecen como una actividad fuera del plan de estudios del posgrado. En este aspecto se encontraron opiniones como la siguientes:

- De hecho, se ofrecieron talleres en todos los semestres del posgrado, iniciando con una fase introductoria y aumentando en profundidad y complejidad a lo largo del trayecto del posgrado; pero no aparecen en el programa de estudios.

- Los investigadores se encargan, personalmente, de ofrecer sus experiencias.

- La institución imparte talleres de manera general para todos los posgrados y para los profesores.

- Se promueven cursos y talleres sobre el sistema APA, en algunos doctorados.

- Se ofrecen estos cursos, pero sólo a nivel doctorado; deberían empezar desde la maestría.

- Resulta muy importante realizar cursos o talleres para mejorar la redacción y la publicación de artículos científicos.

- En mi universidad existen talleres de escritura y publicación anual, principalmente para estudiantes de posgrado.

- Contamos con un taller curricular titulado artículo científico.

- Se oferta actualización continua para la escritura y la redacción de artículos científicos en el área. 
De los 25 académicos que ofrecieron su opinión en torno a la no existencia de estos cursos, se pudo recoger lo siguiente:

- No se ofrecen porque no hay prioridad en la publicación, sólo en terminar la tesis y graduarse.

- Se han tenido varios intentos, pero no hay continuidad.

- No ofrecer estos talleres constituye un grave error de la institución, lo cual no permite mejorar las publicaciones.

- Sólo se ofrecen algunas asesorías acerca de la tesis, pero no existe ninguna invitación a publicar.

- En una ocasión se impartió un curso, pero no está en la curricula; debió haber sido un taller para concluir con la publicación de un artículo en revista indizada.

- El programa considera que cuando terminamos el doctorado ya tenemos todos los elementos para la publicación, pero eso no es así.

Entre las dificultades para publicar, los profesores argumentan, principalmente, la falta de tiempo, esto por las siguientes razones:

- Carga académica excesiva distribuida en tutorías, gestión académica, revisión de programas de la asignatura, revisión y actualización de laboratorios.

- Arguyen que son contadores, psicólogos, etcétera, y atienden las labores domésticas.

- Muchas funciones por atender, las cuales suelen no estar agendadas y son pedidas como urgentes.

- Reducido apoyo por parte de las instituciones al tener carga completa en aulas y no disponer de tiempo para investigación y publicación; esto también influye para no desarrollar capacidades en la publicación.

- Excesiva carga en la docencia que genera el efecto Burnout y disminuye tiempo de ocio para hacer ciencia.

- A los profesores se les carga con demasiadas actividades administrativas, lo cual disminuye el tiempo para publicar.

- Desvió de la atención de la investigaciones para atender evaluaciones de sistemas institucionales tales como el SNI, el Programa de Desarrollo Profesional (Prodep) y el sistema de estímulos institucionales; en todos los casos son formatos diferentes e información diferente.

- Falta de tiempo y apoyo para que los profesores de asignatura desarrollen investigaciones que sean publicables.

Otro grupo se refiere a la falta de capacidad técnica, donde se anota:

- Poco conocimiento sobre la correcta redacción del artículo.

- Carencia de experiencia para moverse en las marañas de la publicación. 
- Inseguridad por la falta de una asesoría eficaz sobre cómo y dónde publicar por parte de un departamento que tenga esta tarea específica.

- No todos los profesores han sido formados y capacitados para generar conocimiento científico; algunos son buenos docentes, pero no participan en las tareas de investigación.

- En la actualidad una limitante es el idioma, que aunque el profesor sea bilingüe; es difícil ser un experto para publicar en inglés, que es en el idioma que se pide en la publicación, con lo cual se observa la discriminación hacia el idioma español.

- Falta de reconocimiento de parte de los índices para algunas revistas, que aunque tienen calidad, las discrimina el Conacyt y otros índices, por lo tanto no tienen validez en el SNI.

- No se tienen los apoyos para impulsar la redacción en inglés, por tanto, tampoco se cuenta con revisores en este idioma; también falta de servicios de traducción especializada.

- Retraso en los tiempos de publicación, al no conocer las dinámicas que siguen la revistas científicas.

- La difícil tarea de pasar los filtros de evaluación de las revistas indexadas, sobre todo cuando el investigador se inicia en la tarea de publicar.

- Limitación de propuestas de artículos científicos adecuados y acabados de parte de los tesistas de doctorado.

Hubo un tercer grupo que sus opiniones las centraron en la falta de apoyo económico, donde sobresale:

- La escasez de recursos para realizar la investigación y publicar resultados; no se cuenta con financiamiento para ninguna de estas actividades.

- La publicación se convierte en un esfuerzo del investigador con los recursos que pueda.

- El factor económico se convierte en una gran limitante para la investigación, pero también la carencia de alumnos interesados que participen en la investigación y en la posible publicación.

- No existen recursos económicos para pagar los costos de la publicación.

- Altos costos de las publicaciones en las revistas.

- Recursos económicos escasos para la adquisición de software.

- Falta de apoyo económico para traducciones, impresión de libros y evaluación de artículos científicos.

- Las contrataciones no son exclusivas para hacer investigación; falta de personal académico que permita atender mayor número de estudiantes, y falta de recursos para realizar mayor cantidad de experimentos que permitan tener resultados exigidos en la actualidad.

Cuando se les pregunta a los investigadores acerca de la medidas para mejorar la publicación de artículos en su institución, el 90\% de ellos hicieron sugerencias, de las cuales el $30 \%$ se refiere a los aspectos económicos, donde se destaca: 
- La preocupación de los profesores para lograr una mayor apoyo para la investigación y no sólo la publicación, ya que si se obtienen buenos resultados en la investigación, la publicación se logra con mayor facilidad.

- Disponer de recursos para hacer los pagos en algunas revistas que cobran por publicar; esto se puede obtener sólo vía Prodep para aquellos que pertenecen a cuerpos académicos.

- Obtener más becas para los estudiantes y mejorar los incentivos para publicar.

- Lograr más apoyos para asistencia a congresos.

- Aprovechar de mejor manera algunas convocatorias, ya que en el Programa Integral de Fortalecimiento Institucional (PIFI) existe un apartado que se denomina "apoyo a publicaciones", pero en ocasiones no se consideran estos fondos.

- Apoyo económico para bases de datos.

- Impulsar el fortalecimiento de las convocatorias internas a proyectos de investigación.

En segundo lugar, aparece la necesidad de impulsar la formación de los académicos en el campo específico de la publicación. Los investigadores opinan que las instituciones deberían:

- Mantener de forma permanente las asesorías y talleres para mejorar los indicadores de publicación de los académicos, así como la revisión constante de trabajos por parte de asesores capacitados.

- Que los asesores de tesis ayuden a mejorar la posibilidad de publicación de los trabajos doctorales.

- Es importante también que se mantengan cursos de redacción con la finalidad de mejorar la presentación de los trabajos de investigación.

- Establecer un diplomado en educación continua para fortalecer la redacción y la presentación de los reportes de investigación.

- La universidad debería contar con un cuerpo editorial que de forma constante se pueda consultar antes de mandar un trabajo a una revista.

- Ofrecer talleres de redacción en el idioma inglés, además de contar con traductores que apoyen en la publicación de trabajos en inglés.

- Introducir una materia formal en los programas de doctorado que ayude en la formación académica para la publicación de artículos.

- Ofrecer cursos de manejo de bases de datos.

- Mejorar la asesoría personalizada en estas actividades.

- Mejorar los cursos de formación metodológica, y obtener un mayor provecho de las estancias académicas.

El tercer punto en importancia se ubicó la infraestructura científica e institucional, donde los investigadores opinaron que: 
- Las universidades deben promover la formación de redes con la finalidad de mejorar sus políticas de difusión de la ciencia.

- Las autoridades universitarias necesitan mejorar su contacto con los investigadores formando los organismos y mecanismos necesarios para ello.

- Definir mecanismos para incentivar la escritura científica y la publicación.

- Crear los grupos de investigación que promuevan la publicación en la institución.

- Definir reglas e instrumentos para fortalecer el trabajo colaborativo.

- Que las medidas de impulso a la publicación lleguen a todos los campos de la universidad, que no se concentren en unas áreas y en el campo central.

- Definir cuerpos académicos para la asesoría y el impulso a la producción de artículos científicos.

- Promover la producción con la participación de varios cuerpos académicos.

- Mejorar los espacios físicos para los investigadores.

- Mejorar la conexión y la relación entre las coordinaciones de posgrado, con las de investigación y con los investigadores; evitar los espacios ocupados por personal que no cumple con el perfil, que no saben qué hacer con la investigación y el posgrado.

- Mejorar los espacios para el trabajo de investigación, y asignar personal de apoyo administrativo para las actividades de gestión académica, lo cual permita liberar tiempo y destinarlo a la actividad de investigación.

- Se puede observar un desequilibrio entre las instituciones en cuanto a estas actividades, pues comparando entre éstas, e incluso entre facultades, existe una gran diferencia; en algunas de ellas se cuenta con apoyo muy importante para las personas que desean publicar, pues entre las políticas más relevantes es posible mencionar (en algunas universidades y facultades): a) financiamiento para gastos de publicación, b) disminución de horas clase para investigadores con alta producción académica y estímulos económico, c) acceso a bases de datos de primer nivel, y d) presupuesto para equipamiento y compra de reactivos.

Otro punto fue el de las políticas de difusión donde los investigadores recomiendan:

- Mejorar las redes entre universidades con la finalidad de impulsar las coediciones.

- Definir la política de las revistas, ya que se envían artículos y no se responde a los profesores que no saben cuáles son las normas de publicación de autores pertenecientes a la misma institución; se dice que las publicaciones deben ser externas y no internas, pero no existe normatividad al respecto.

- Brindar mayores oportunidades de publicar a los profesores jóvenes, ya que los consolidados no tienen dificultad para ello.

- Continuidad a las revistas universitarias. 
- Fortalecer los programas editoriales de las universidades: cuidar mejor la edición de los libros universitarios, porque, generalmente aparecen con errores.

- Mayor claridad en cómo se miden las publicaciones, de cuáles son los factores que cuentan para su medición.

- Que las convocatorias a los talleres de publicación se hagan en forma abierta y no solamente se invite a algunos profesores.

En cuanto a la última pregunta que se refiere a si los investigadores conocen una revista dónde publicar sus trabajos, se levantó una lista de 334 distribuidas por áreas del conocimiento, según la taxonomía del Conacyt, quedando de la forma siguiente:

- Área I. Físico matemáticas y ciencias de la tierra, 25 revistas

- Área II. Biología y química: 44

- Área III. Ciencias Médicas: 30

- Área IV. Humanidades y ciencias de la conducta: 63

- Área V: Ciencias Sociales: 120

- Área VI. Biotecnología y ciencias agropecuarias: 8

- Área VII. Ingenierías: 20

- Interdisciplinarias: 6

Además, se contó con 18 revistas que no se pudieron ubicar en ninguna área de conocimiento al no ser localizadas en la Web.

En México los investigadores están obligados a buscar revistas en el extranjero, lo cual se deriva del reducido número de publicaciones mexicanas reconocidas en los índices de mayor influencia. El registro que tenía México en scopus en 2016 era de 103 revistas, 50\% de éstas ubicadas en el cuartil 4, $40 \%$ en el 3, 10\% en el 2 y ninguna en el 1. El listado de revistas incluidas en el Conacyt comprendía, en el mismo año, 137, por lo que realmente el espacio para publicación en México es reducido. Se debe considerar que el SNI integra a un poco más de 27000 investigadores, y que la publicación científica en este país depende del SNI, pues al realizar un ejercicio de correlación entre el número de artículos publicados por mexicanos a nivel mundial con el crecimiento del SNI, entre el año 2000 y 2015 se encontró que es casi igual a 1. En tal situación, los investigadores se ven obligados a publicar en el extranjero o a buscar revistas nacionales que no se inscriben en el JCR, o en el listado del Conacyt, sobre todo los investigadores jóvenes, aunque algunos evaluadores las denominan revistas "tóxicas", lo cual es injusto, ya que algunas de ellas han alcanzado buenos reconocimientos, sobre todo en SciELO. 


\section{Conclusiones}

México ha incrementado su participación en ciencia mundial en cuanto al número de artículos científicos publicados por investigadores mexicanos, pero este incremento no se observa en la misma dimensión en cuanto al mejoramiento de los indicadores de penetración de los contenidos científicos medidos a través del índice $\mathrm{H}$, el Factor de Impacto y el Impacto Relativo al Mundo. Los investigadores manifestaron que una adecuada difusión de la ciencia no es una política que se impulse en las universidades, pues realizaron una serie de elementos y carencias que no permiten llevar a cabo las labores de investigación y difusión de la ciencia en forma adecuada. Las instituciones relacionadas con la investigación deben preocuparse, también, por fortalecer la difusión de los resultados de dicha investigación y por mejorar la calidad de las publicaciones. A pesar de ser muy limitada esta preocupación, las publicaciones científicas de los investigadores mexicanos han logrado incrementarse en el contexto mundial y con ello mejorar la posición de México.

En la encuesta se pudo encontrar una preocupación de los académicos por impulsar el mejoramiento de la ciencia, así como el posicionamiento académico de las instituciones y programas académicos donde laboran. Lo anterior no es acorde con la opinión de que los investigadores mexicanos trabajan sólo para mejorar el puntaje en las evaluaciones y buscan reunir documentos para mejorar en los diferentes sistemas de evaluación.

Entre las dificultades para publicar, lo investigadores insistieron, principalmente, en la falta de tiempo para hacer investigación. En las instituciones de educación superior es una realidad que, ante las reducciones presupuestarias, los directivos de las facultades buscan que los profesores de tiempo completo se carguen con un mayor número de horas clase e incrementan los compromisos administrativos, lo cual va en detrimento del tiempo dedicado a la investigación. La disminución del tiempo a la investigación afecta los posibles talleres de entrenamiento metodológico y técnico para mejorar la investigación, y con ello las publicaciones tanto en su cantidad como en la calidad. El 90\% de los investigadores incluyeron el factor económico como el central para el impulso a la investigación en México, y esto deriva en el debilitamiento de los mecanismos para la difusión de la ciencia.

Pero también se debe fortalecer el aspecto institucional de las universidades mediante el impulso de redes de cooperación para el desarrollo científico y fortalecer la capacidad de difusión de la ciencia; promover la preparación para comunicar los resultados de investigación desde el posgrado, para que los estudiantes puedan publicar artículos de sus resultados de investigación. 
Al final, los investigadores dieron a conocer el nombre de 334 revistas, muchas de ellas del extranjero y algunas no reconocidas por el índice del Conacyt, ya que este listado de revistas sólo reconoce a 137 publicaciones, lo cual constituye un número muy reducido para dar posibilidades de publicación a los más de 27000 investigadores que comprende el sNI. Se debería incrementar en número de revistas de calidad en nuestro país

\section{Referencias}

American Psychological Association (2010a). Publication Manual of the American

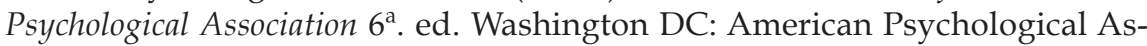
sociation.

American Psychological Association (2010b). Manual de publicaciones de la American Psychological Association 3a. ed. México: Manual Moderno

Belcher, Wendy L. (2009). Writing your journal article in 12 weeks. A guide to academic publishing success. Thousand Oaks: Sage Publications Inc.

Bost, Holst (2015). Scientific paper writing: a survival guide. Bergen: University of Bergen

Cargill, Margaret y O'Connor Patrick (2009). Writing scientific research articles. Strategy and steps. West Sussex: Wiley-Blackwell.

Claudio, Luz (2016). How to write and publish a scientific paper: The step-by-step guide. San Juan: Write Science Now Publishing Co.

Consejo Nacional de Ciencia y Tecnología (Conacyt) (2015). Informe general del estado de la ciencia, la tecnología y la innovación. México: Conacyt. Recuperado de: www.siicyt.gob.mx/index.php/estadisticas/informe-general/informegeneral-2015/3814-informe-general-2015/file

Consejo Nacional de Ciencia y Tecnología (2017a). Reglamento del Sistema Nacional de Investigadores. Recuperdo de: https://www.conacyt.gob.mx/index.php/ el-conacyt/sistema-nacional-de-investigadores / marco-legal/ reglamentosni/13493-reglamento-sni/file.

Consejo Nacional de Ciencia y Tecnología (Conacyt) (2017b). Criterios SNI. Recuperado de: https://www.conacyt.gob.mx/index.php/el-conacyt/sistemanacional-de-investigadores/marco-legal/criterios-sni.

Day, Robert y Gastel, Barbara (2006). How to write and publish a scientific paper, 6 . ed. Wesport: Greenwood Prees.

Debnath, J. y Venkatesh, M.D. (2015). Writing and publishing a scientific paper: Facts, myths and realities. Medical Journal Armed Forces India (MJAFI), 72(2), 107111.

Difabio de Aglat, Hilda (2011). Las funciones del tutor de la tesis doctoral en educación. Revista Mexicana de Investigación Educativa, 16 (50), 935-959.

Dominguez-Montanari, Sophie (2010). How to write and publish a scientific research paper, Science for all. Recuperado de: http:/ / scienceforall.org. 
Espino-Datsira, Sandra (2015). La enseñanza de estrategias de escritura y comunicación de textos científicos y académicos a estudiantes de posgrado. Revista Mexicana de Investigación Educativa, 20(66), 959-976.

Gomez-Nashiki, Antonio; Jiménez-García, Sara Aliria y Moreles-Vázquez, Jaime (2014). Publicar en revistas científicas, recomendaciones de investigadores de ciencias sociales y humanidades. Revista Mexicana de Investigación Educativa, 19 (66), 155-185.

Katz, Michael J. (1985). Elements of the scientific paper. A step by step guide for students and professionals. New Haven y London: Yale University Press.

Lindholm, Jennifer A., Szelényi, Katalin; Hurtado, Sylvia, y Korn, William S. (2005). The American College: National Norms for the 2004-2005 HERI Faculty Survey. Los Angeles: UCLA Higher Education Research Institute.

Lopez-Leyva, Santos (2013). El proceso de escritura y publicación de un artículo científico. Revista Electrónica Educare, 17 (1), 7-27.

Ortinau, David J. (2011). Writing and publishing important scientific articles. A reviewer's perspective". Journal of Business Research, núm. 64, 150-156

Sabaj, O. (2009). "Descubriendo algunos problemas de la redacción de artículos de investigación científica de los alumnos de postgrado. Revista Signos, 42 (69), 107-127. 